\title{
Simulation of Gas Water Two-phase Flow in Diesel Turbocharger
}

\author{
J. Yao ${ }^{1}$, Y. Yao ${ }^{1 *}$, P.J. Mason ${ }^{1}$, T. Zhang ${ }^{1}$, F.J.G. Heyes ${ }^{2}$, P.E. Roach ${ }^{2}$ \\ 1: Faculty of Engineering, Kingston University, London SW15 3DW, UK, y.yao@ kingston.ac.uk \\ 2: Napier Turbochargers Ltd., Waterside South, Lincoln LN5 7FD, UK
}

\begin{abstract}
Numerical simulation of a gas water two-phase flow in Diesel Turbocharger has been carried out using computational fluid dynamics solution of the Eulerian Reynolds-averaged Navier-Stokes equations for the continuum gas phase and the Lagrangian particle tracking method for the discrete water droplets. A generic diesel turbocharger configuration was chosen, which has an upstream duct inlet and a downstream blade ring outlet. Three identical water injectors were evenly distributed in the circumferential direction and located upstream of the blades. Simulation considered water injection at an angle of $30^{\circ}$ from the centerline with two water pump pressures of 4 bars and 8 bars. The process of liquid droplet break-up has been modeled using the Blob model for primary break-up and the cascade atomization breakup (CAB) and the Reitz and Diwakar breakup (RDB) models for secondary break-up. The results show that the predicted water droplet coverage and the blade temperature drop were in good agreement with experimental measurements. Simulations also showed that for the two water pump pressures considered, the water droplet coverage and distribution patterns on the blade ring change little, indicating the need to increase the number of injectors for better water washing performance.
\end{abstract}

Keywords: Two-phase Flow Simulation, Water Droplets, Turbocharger cleaning

\section{Introduction}

Turbocharged diesel engines are in high demand by industry and the trend of reducing operating cost has driven engine operators to use more economic fuels, e.g. low grade heavy duty diesel. This leads to deposits accumulating inside the engine system, especially around the stator blade ring in a turbocharger. Because of this, the turbocharger performance will be degraded considerably with lower working capacity, efficiency and engine power outputs. To remove these deposits, industry often uses two cleaning methods: 'dry' cleaning by injecting small-medium sized nut shells and 'wet' cleaning by injecting water liquid. These methods were initially developed for cleaning compressor blade [Mund and Pilidis, 2004], and now have successfully been extended for cleaning turbocharger blade. Some developments have shown that the trend of using the online 'wet' cleaning has been increased due to the wide availability of water at low cost. To reflect this, the present study is an investigation of water washing for turbocharger blade deposit removal, while the engine system is still under normal operation conditions (i.e. online).

In principle, it is a two-phase flow problem with a mixture of 'hot' gas and 'cold' water. As the gas mixture flow is governed by conservation laws of compressible fluids, the introduction of incompressible water liquid droplet will bring additional difficulties for numerical simulations, along with the complicated droplet break-up at near the injector. Using in-house experiments can provide some information such as temperature distributions on the blade ring by traditional thermocouple measurements, however in-depth flow features are difficult to obtain. Computational fluid dynamics simulation could therefore be a useful tool to provide quantitative and qualitative predictions of this complicated two-phase flow problem [Ryan, 2006], such that the gas mixture flow, the water liquid trajectory and the other important interactions between these two phases can be properly captured by using suitable numerical 
schemes and treatments to solve the governing RANS equations. Among various methods, the Euler-Lagrangian approach has been increasingly adopted. In this approach, the continuum gas mixture flow is solved by the RANS equations in the Eulerian frame, and the discrete liquid particle trajectories are tracked in the Lagrangian frame. A previous study showed that it can overcome some numerical difficulties existing in other methods [Aktas and Mahaffy, 1996].

Numerical studies have been performed for a single-phase gas mixture flow in turbocharger [Yao et al. 2007] and a two-phase gas-water flow with the water injected in alignment with the gas flow direction [Yao et al. 2008]. The present work continues the two-phase modeling study by considering the water injected at an angle of $30^{\circ}$ from the gas flow direction. The corresponding experimental tests were carried out on an in-house turbocharger test rig at Napier Turbocharger Ltd. with the blade temperature measurements at two water pump pressures of 4 and 8 bars. The CFD study is performed with a two-way coupling treatment. As the gas mixture flow field seems very buoyant because of the density difference between the two phases, the proportional mass friction of the water vapor in the gas mixture has shown strong dependency on the water mass flow rate injected into the domain and the subsequent liquid evaporation process. These will be considered by the Antoine equation that deals with the thermal coupling of the two phases. The water droplet break-up process is treated in two stages: (1) a primary break-up using the Blob model, which provides initial droplets size and the penetration length; (2) a secondary break-up using both the cascade atomization break-up (CAB) model and the Reitz and Diwakar break-up (RDB) model, which determine the break-up mode, the water droplet sizes and the distributions along the trajectory path. The predicted water droplet distribution and the temperatures on the blade ring will be directly compared with the available experimental measurements, and certain details of droplets characteristics will also be analyzed.

\section{Numerical Methods}

\subsection{Governing equations}

The continuum phase of the gas mixture flow is governed by three-dimensional compressible Navier-Stokes equations presented in vector form as

$$
\begin{aligned}
& \frac{\partial \rho}{\partial t}+\nabla \bullet(\rho U)=0, \\
& \frac{\partial(\rho U)}{\partial t}+\nabla \bullet(\rho U \otimes U)=-\nabla p+\nabla \bullet \tau+S_{M}, \\
& \frac{\partial\left(\rho h_{t o t}\right)}{\partial t}-\frac{\partial p}{\partial t}+\nabla \bullet\left(\rho U h_{t o t}\right)=\nabla \bullet(\lambda \nabla T)+, \\
& \nabla \bullet(U \bullet \tau)+U \bullet S_{M}+S_{E}
\end{aligned}
$$

where $\rho$ is the density, $U$ is the velocity vector, $p$ is the pressure, $\tau$ is the shear stress tensor, $h$ is the enthalpy, $\lambda$ is the heat conductivity coefficient, $T$ is temperature, and $S_{M}$ and $S_{E}$ are sources terms for the momentum and energy equations respectively.

\subsection{Lagrangian particle tracking}

In this method, the displacement of a tracked droplet (particle) along its trajectory is calculated by the integration of droplet velocity over a time step and at the end of the time step, the droplet velocity is then updated as

$$
\begin{aligned}
& x_{i}^{n}=x_{i}^{o}+u_{p i}^{o} \delta t, \\
& u_{p}=u_{f}+\left(u_{p}^{o}-u_{f}\right) \exp \left(\frac{\delta t}{t_{c}}\right), \\
& +t_{c} F_{\text {all }}\left(1-\exp \left(-\frac{\delta t}{t_{c}}\right)\right)
\end{aligned}
$$

where $x$ is the coordinates, $u$ is the velocity vector, $\delta t$ is time step, $t_{c}$ is the characteristic time scale, $F_{\text {all }}$ is interaction force. Superscripts ' $n$ ' represents a new location, and ' $o$ ' an old location. Subscripts ' $i$ ' represents the index of particles, ' $p$ ' the particle and ' $f$ ' the fluid, respectively.

\subsection{Euler-Lagrangian Coupling}

At each time step, the fluid properties (i.e. density, viscosity and velocity) at the current location of a particle are required to accurately calculate the next movement of the particle. Due to the fluid-particle interactions, the particle motion will also be influenced by some other factors, e.g. the viscous drag, the velocity difference and the particle size, etc. For a dense 
particle cluster in the continuous fluids as seen in present case, the flow and the particle interactions can be better presented in a twoway coupling system, where the effect of particles can be implemented by source terms in the momentum and energy equations, and particle movements can be tracked using equations (4) and (5). The ANSYS-CFX v11 software has been used for the simulation.

\subsection{Liquid evaporation model}

The liquid evaporation model is activated to simulate heat and mass transfer of the water droplets in a hot gas mixture environment. The mass re-distribution in a two-phase flow system is determined by the Antoine equation on the basis of water vapor pressure, defined as

$P_{\text {vap }}=P_{\text {ref }} \exp \left(A-\frac{B}{T+C}\right)$,

where $P$ and $T$ are pressure and temperature, and $A, B, C$ are the default Antoine coefficients.

Based on the liquid boiling temperature and the particle temperature, the following treatments are considered: for water droplet temperature lower than the boiling temperature, the mass transfer is mainly convective; and for water droplet temperature higher than the boiling temperature, the mass transfer follows the formula as

$$
\frac{d m}{d t}=\pi d D S h \frac{W_{C}}{W_{G}} \log \left(\frac{1-X}{1-X_{G}}\right),
$$

where $W_{C}$ and $W_{G}$ are the molecular weights of the water vapor and the gas mixture in the continuous phase, while $X$ and $X_{G}$ are the molar fractions of the particle phase and the gas phase, respectively.

\subsection{Liquid break-up models}

The water atomization process in the vicinity of the injector exit is described by the Blob method, which provides a description of the primary water droplet break-up without the atomization details. In brief, the water droplet is assumed to be initially in a spherical shape with a diameter, equivalent to that of the water nozzle injector, i.e. $D_{p}=D_{\text {nozle }}$. Immediately after injecting into the gas mixture field and with the Webber number greater than the critical value, the secondary break-up occurs. Among various available models, the Reitz and Diwakar break-up (RDB) model [1987] and the cascade atomization break-up (CAB) model [Tanner, 2003] are often used. Note that the RDB model was originally proposed for stripping (shear layer) break-up mode, and the $\mathrm{CAB}$ model is an extension of the standard enhanced Taylor Analogy Break-up (ETAB) model [Tanner, 1998].

\section{Experimental Tests}

NAPIER turbochargers are typically designed for operating at both part-load and full load conditions. The configuration of a complete turbocharger is rather complicated and in order to focus on the two-phase flow study, we adopted a simplified geometry as seen in Fig. 1, which consists of a bent duct to guide the inlet gas mixture from the engine exhaust, three guide struts and a central cone, and a blade ring of 24 nozzle vanes evenly distributed in the circumferential direction downstream. The Reynolds number is about $1 \times 10^{6}$ based on the duct diameter and the inflow gas mixture velocity. Hence, the flow is turbulent and the standard two-equation k- $\varepsilon$ model is used to determine the turbulent viscosity and the eddy dissipation rate.

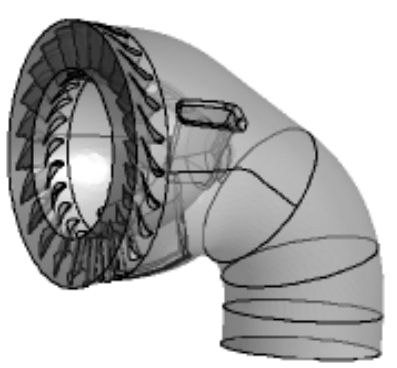

Figure 1: Sketch of configuration for modelling.

A total of three single-hole water injectors are installed at a short distance upstream of the blade ring. One injector nozzle is placed at the top (i.e. the maximum height location in the cross plane), and the other two are symmetrically located at $120^{\circ}$ in both clockwise and anti-clockwise directions. The injector inlet is $6 \mathrm{~mm}$ in diameter and the water injection is aligned in an angle of $30^{\circ}$ against 
the cross gas flow direction. The injector finishes with a hemisphere shape, and the length to the diameter ratio is above 10 . In the experiment the water, at the room temperature, is introduced to the water injectors from a pressurized water tank at a pump pressure of 4 $\sim 8$ bars. A group of thermocouples are preinstalled at the leading-edge of the blades. Before washing starts, the turbocharger is operated at a condition of $900 \mathrm{~N} / \mathrm{T}^{0.5}$, where $\mathrm{N}$ is the rotor speed in rpm and $\mathrm{T}$ is the turbine inlet temperature in Kelvins. The measured blade temperatures are about 520 Kelvin during operation. After sufficient water washing time (typically in an order of 20 seconds), those blades that have been 'washed' will have noticeable temperature decreases, indicated by the thermocouple measurements. In practice, tests are repeated several times in order to minimize the uncertainty of the test data.

\section{Results and Discussions}

The computational domain is discretised using a hybrid meshing technique (i.e. hex/prism meshes in the near wall and tetrahedral meshes in the far field) and the governing equations are solved by the finite volume method. The mesh density and its distributions are carefully generated with finer grids clustered around regions where the geometry and the flow change rapidly, e.g. near the water injector exits and around the blades. The boundary conditions are as follows; a total pressure from test is set at the inlet and at the outlet, a static pressure is tuned to satisfy the test inlet mass flow rate. The usual no-slip wall condition is used for solid walls together with the adiabatic thermal condition. Similar to that in the experiment, simulation of a single-phase gas mixture flow is carried out first to build up a steady flow field, and then a two-phase flow modeling follows. A total of 30,000 water particles are injected into the flow domain via three water injectors (i.e. 10,000 for each water injector) and the injections are kept in an uncorrelated manner.

The mesh-independence study has been performed on coarse and fine meshes with a grid of 1.5 and 2.3 million nodes, respectively. The mesh resolutions are fairly good in the near-wall $\left(\mathrm{y}^{+}=5\right)$, extending to $\mathrm{y}^{+}=35$ for the finer mesh and $\mathrm{y}^{+}=112$ for the coarse mesh. Fig. 2 gives the variations of liquid water droplets temperature as a function of diameter. Note that due to the use of the Blob model, the droplets start with the injector diameter of 6 $\mathrm{mm}$ at the room temperature of $298 \mathrm{~K}$. Immediately after the injector, the droplet sizes decrease rapidly down to a scale of $10^{-5} \mathrm{~mm}$ due to the secondary break-up mechanism (atomization). After this, the droplet size remains unchanged with temperature increased to about $350 \mathrm{~K}$. Results from two simulations are very similar; with small discrepancies observed at the end of the secondary break-up. It seems that using the finer grids in the vicinity of the injector exit will further enhance the secondary break-up. As the finer mesh improves the simulation both near injectors and around blades, the remaining simulations are all based on this mesh.

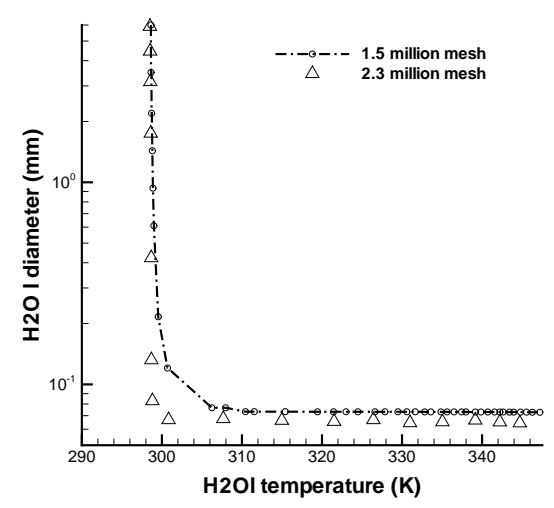

Figure 2. Variation of the liquid water droplet temperature against the diameter.

Simulation validation was carried out on the comparison of blade temperature measurements at water pump pressures of 4 bars and 8 bars. At an operation speed of $900 \mathrm{~N} / \mathrm{T}^{0.5}$, the blade temperature seems to maintain almost constant about $515 \sim 525{ }^{\circ} \mathrm{C}$ before the water washing. This agrees with previous studies by the present authors [Yao et al. 2007]. After injecting water liquid at ambient room temperature of $25{ }^{\circ} \mathrm{C}$, some un-evaporated water droplets will travel on to the blade surfaces, causing the blade wall temperature decreases due to the heat transfer. Fig. 3 displays the blade temperature variations from experimental measurement and numerical 
prediction at a water pump pressure of 4 bars. It can be seen that considerable temperature decreases to about $70 \sim 100^{\circ} \mathrm{C}$ appear in three areas. Not surprisingly, those 'unwashed' blades exhibit a slightly increased surface temperatures. This is mainly due to additional heat supplied to the turbocharger by the control system of the turbocharger test rig when cold water is added to the system.

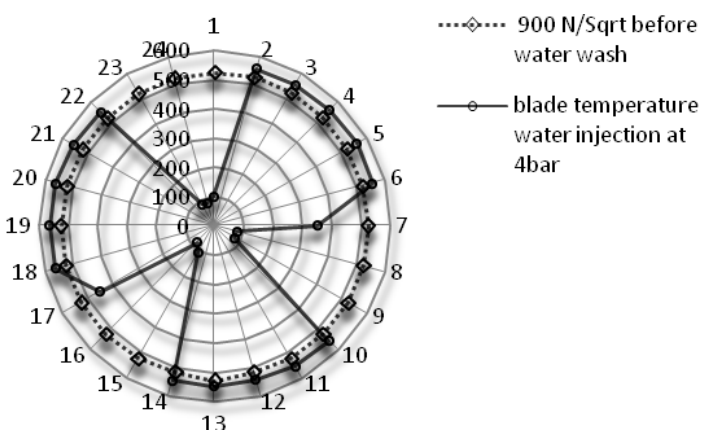

Figure 3. Test water coverage at pump pressure of 4 bars: the blade number in circumference direction and the measured temperature $\left({ }^{\circ} \mathrm{C}\right)$ in radial direction.

Figure 4 gives the comparison of the CFD predicted water coverage by using the $\mathrm{CAB}$ model and the RDB model, respectively. It can be seen that while two models produce quite similar results compared to the test results, the RDB model gives slightly wider water coverage. The reason of this over-prediction is probably due to the shear layer break-up mode in the RDB model that may lead to larger spreading angle, then covering wider area in downstream. It is thus concluded that for this problem, the CAB model prediction is better in agreement with the test. Simulations also show that the droplet temperatures are in a range of $298 \mathrm{~K}$ to $364 \mathrm{~K}$. Although there are no experimental data to compare, simulation does reveal that considerable water evaporation happens when the temperature difference between the blade surfaces and the surrounding fluids reaches about $161 \mathrm{~K} \sim 227 \mathrm{~K}$ range.

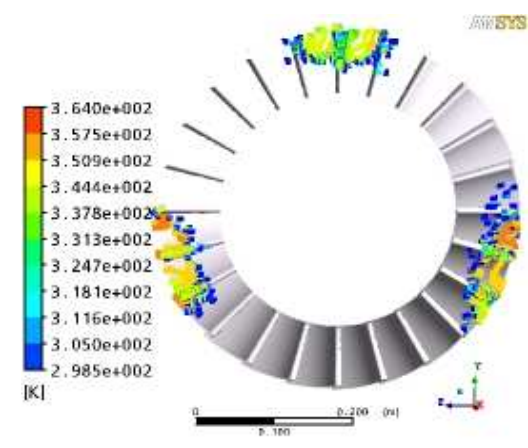

(a)

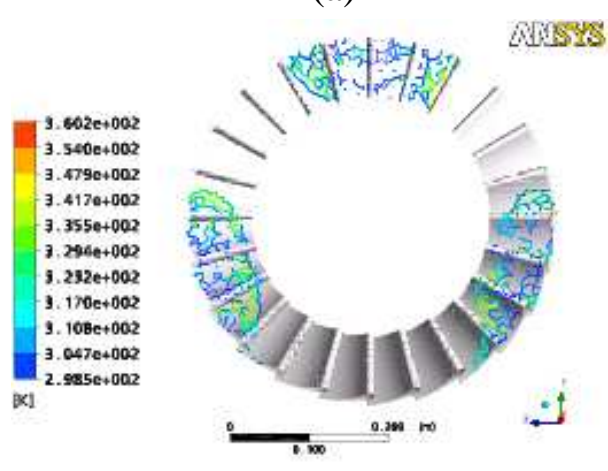

(b)

Figure 4. CFD predicted water coverage. (a) $\mathrm{CAB}$ model; (b) RDB model.

Simulation continues for a water pump pressure of 8 bars and Figure 5 gives the test results. At this condition, there is no significant difference observed in comparison to that of a water pump pressure of 4 bars. Note that thermocouple at blade 7 failed to respond in the precursor no water injection tests. After the water washing, about 12 blades have been washed with decreased surface temperature. As the CAB model gives better predictions from previous analysis, here simulation only uses this model. Figure 6 gives the predicted water coverage. Comparing to the measurement, the $\mathrm{CAB}$ model predictions at water pump pressures of 4 bars (see Fig. 4a) and 8 bars (see Fig. 6) are quite similar in terms of the pattern of water droplets on the blade ring. Data analysis continues on the correlation of droplet sizes with the temperature. Figure 7 depicts the comparisons of simulation results. It can be seen that the two $\mathrm{CAB}$ predictions agree very well, despite of a factor of 2 in the water pump pressure. The prediction of RDB model shows 
considerable difference in the area when water droplet breaks up to the smallest scale, indicating different mechanism applied.

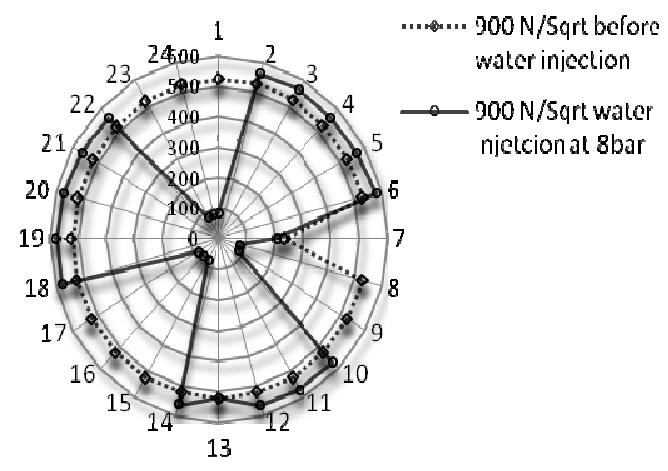

Figure 5. Test water coverage at pump pressure of 8 bars: the blade number in circumference direction and the measured temperature $\left({ }^{\circ} \mathrm{C}\right)$ in radial direction.

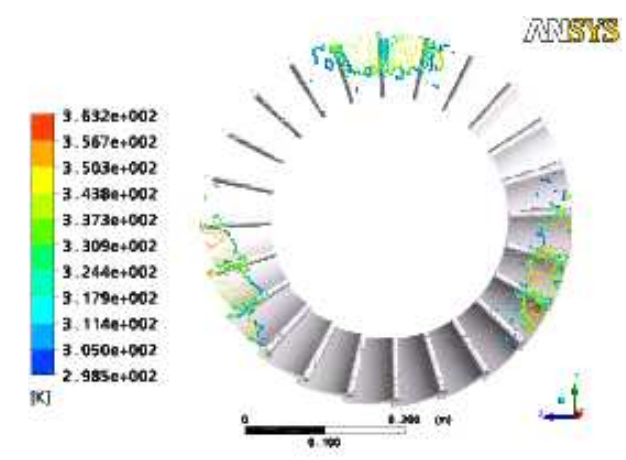

Figure 6. CFD predicted water coverage at 8 bars pump pressure using $\mathrm{CAB}$ model.

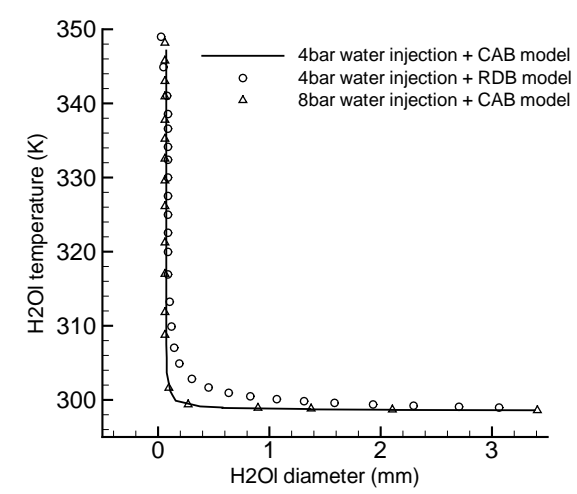

Figure 7. Comparisons of water droplets temperature and its diameter.

\section{Conclusions}

Numerical study of a gas water two-phase flow in a turbocharger configuration has been carried out by solving the RANS equations for the continuum gas phase, and the particle tracking method for the discrete water droplets. A two-way coupling is considered with the water evaporation model and the droplet breakup models for fluid and thermal exchange between two phases. Simulations have been performed at two test conditions of water pump pressures of 4 bars and 8 bars, and the predicted water coverage using the $\mathrm{CAB}$ model is in good agreement with the measurements, while the results of the RDB model slightly over-predict. Consistent with test results, the CFD predictions also illustrate that by increasing the water pump pressure from 4 bars to 8 bars; there is negligible impact on the water coverage, indicating the necessary of increasing the number of water injectors rather than the water pump pressure.

\section{References}

Aktas, B. and Mahaffy, J.H., 1996. A two-phase level tracking method, Nuclear Engineering and Design, 162: 271-280.

Mund, F.C. and Pilidis, P., 2004. A Review of Gas Turbine On line Washing System, ASME GT-2004-53224.

Reitz, R.D. and Diwakar, R., 1987, Structure of high-pressure fuel sprays, SAE 1987-05-98.

Ryan, M.J., 2006. CFD prediction of the trajectory of a liquid jet in a non-uniform air crossflow, Computer \& Fluids, 35:463-476.

Tanner, F.X., 1998. Liquid Jet Atomization and Droplet Breakup Modeling of Non-evaporating Diesel Fuel sprays, SAE Technical Paper 970050. Also in SAE 1997 Transactions: Journal of Engines, 106(3): 127-140.

Tanner F.X., 2003. A Cascade Atomization and Drop Breakup Model for the Simulation of HighPressure Liquid Jets," SAE Paper 2003-01-1044.

Yao, J., Yao, Y., Mason, P.J., Zhang, T., Heyes, F.J.G., Roach, P.E., 2007, Computational study of flow structure and energy loss in turbocharger, The Fifth International conference on fluid mechanics (ICFM-V), Shanghai China, Springer-Verlag publisher.

Yao, J., Yao, Y., Mason, P.J., Zhang, T., Heyes, F.J.G., Roach, P.E., 2008, CFD Modelling of Water Injection Flow in Turbocharger, The Fifth International Conference on Computational Fluid Dynamics (ICCFD5), Soul, Korea, Springer-Verlag publisher. 